\title{
Les Facteurs Associés Aux Calcifications Valvulaires Du Cour Et Ou Des Gros Troncs Artériels Chez Les Hémodialysés
}

\section{Fousseny Diakité}

Service néphrologie -hémodialyse Hôpital Aristide Le Dantec (Sénégal), Service néphrologie -hémodialyse Hôpital de Donka (Guinée)

\author{
Mohamed Sidy Seck \\ El Hadji Fary Ka \\ Ahmed Tall \\ Mohamed Moustapha Cissé \\ Abdou Niang \\ Boucar Diouf
}

Service néphrologie -hémodialyse Hôpital Aristide Le Dantec (Sénégal)

Kane Moussa

Dioum Momar

Abdoul Kane

Service de cardiologie Hôpital Général de Grand Yoff (Sénégal)

Doi:10.19044/esj.2021.v17n7p200

Submitted: 25 May 2020

Accepted: 12 June 2020

Published: 28 February 2021
Copyright 2021 Author(s)

Under Creative Commons BY-NC-ND

4.0 OPEN ACCESS

Cite As:

Diakité F., Sidy Seck M., Fary Ka E.H., Tall A., Cissé M.M., Niang A., Diouf B., Moussa K., Momar D. \& Kane A. (2021). Les Facteurs Associés Aux Calcifications Valvulaires Du Cour Et Ou Des Gros Troncs Artériels Chez Les Hémodialysés. European Scientific Journal, ESJ, 17(7), 200. https://doi.org/10.19044/esj.2021.v17n7p200

\section{Résumé}

Introduction: Le but de l'étude était de déterminer la prévalence ainsi que les principaux facteurs de risque associés au développement des calcifications valvulaires du cœur et/ou d'au moins un gros tronc artériel chez les hémodialysés. Patients et méthodes: Etaient inclus les patients qui avaient présenté à l'échodoppler cardiaque une calcification valvulaire du cœur et ou d'au moins un gros tronc artériel. Les variables suivantes avaient été étudiées : épidémiologiques, cliniques et paracliniques. Résultats: Sur 54 patients hémodialysés, 51 avaient été explorés parmi lesquels 39 présentaient des 
calcifications cardio-vasculaires. Les femmes étaient les plus touchées 66.67 $\%$ avec un sex- ratio de $1 / 2$. Les calcifications valvulaires représentaient $64.1 \%$ et les calcifications vasculaires $76.9 \%$. Les atteintes univalvulaires représentaient $68 \%$ suivies des atteintes bivalvulaires $24 \%$ et trivalvulaires $8 \%$. Les calcifications des artères fémorales étaient les plus fréquemment rencontrées $63.3 \%$ suivies des artères iliaques $53.3 \%$. Conclusion: Les calcifications cardiovasculaires sont fréquentes chez les hémodialysés. Les principaux facteurs de risque liés à la survenue des calcifications étaient : l'hypocalcémie, l'hypo et l'hypercholestérolémie à LDL, l'hyper cholestérolémie totale et le taux sanguin de PTHi augmenté. La correction de ces troubles pourrait prévenir la survenue des calcifications.

Mots clés : Calcifications Cardio-valvulaires, Hémodialysés

\title{
Study of Factors Associated With Valvular Calcifications of the Heart and or Large Arterial Trunks in Hemodialysis Patients
}

\section{Fousseny Diakité}

Nephrology - hemodialysis service Aristide Le Dantec Hospital (Senegal), Nephrology - hemodialysis service Donka Hospital (Guinée)

Mohamed Sidy Seck

El Hadji Fary Ka

Ahmed Tall

Mohamed Moustapha Cissé

Abdou Niang

Boucar Diouf

Nephrology - hemodialysis service Aristide Le Dantec Hospital (Senegal)

Kane Moussa

Dioum Momar

Abdoul Kane

Cardiology Department Grand Yoff General Hospital (Sénégal)

\begin{abstract}
Introduction: The purpose of the study was to determine the prevalence and key risk factors associated with the development of valve calcifications of the heart and/or at least one large arterial trunk in hemodialysis. Patients and methods: Patients who had presented with cardiac echodoppler a valve calcification of the heart and or at least one large arterial trunk were included. The following variables had been studied: epidemiological, clinical and paraclinical. Results: Of 54 hemodialysis
\end{abstract}


patients, 51 were explored, 39 of whom had cardiovascular calcifications. Women were the most affected $66.67 \%$ with a sex ratio of $1 / 2$. Valvular calcifications represented $64.1 \%$ and vascular calcifications $76.9 \%$. Univalvular involvement accounted for $68 \%$ followed by bivalvular involvement $24 \%$ and trivalvular involvement $8 \%$. Calcifications of the femoral arteries were the most frequently encountered $63.3 \%$ followed by the iliac arteries 53.3\%. Conclusion: Cardiovascular calcifications are common in hemodialysis patients. The main risk factors related to the onset of calcifications were: hypocalcemia, hypo and hyperlolesterolemia with LDL, hyper hyper cholesterolemia and increased blood PTHi levels. The correction of these disorders would be a way to prevent the occurrence of calcifications.

Keywords: Cardio-Valvular Calcifications, Hemodialysis

\section{Introduction}

Les calcifications cardio-vasculaires (CCV) sont dues aux dépôts de phosphates de calcium au niveau des valves cardiaques et ou des gros troncs artériels. En pratique le diagnostic est posé à l'aide de l'échocœur.(Defrancisco AM et al, 1985).

Les calcifications artérielles et valvulaires sont fréquentes chez les patients hémodialysés. La vitesse de progression des calcifications est plus rapide chez ces patients comparativement à la population générale. Les dépôts calciques sont soit localisés dans la média des parois vasculaires, soit présents au niveau des plaques d'athérome sous-intimales. Ces deux types de dépôts sont souvent observés simultanément chez l'homme, et partagent probablement certains mécanismes moléculaires et cellulaires en ce qui concerne leur genèse (Kalantar-Zadeh K, Kuwae N, Redigor DL, et al ; 2006).

Le développement des complications cardio-vasculaires athéromateuses et/ou calcifiantes chez les patients en insuffisance rénale chronique (IRC) implique la responsabilité de multiples facteurs de risque dont les effets s'additionnent. Les uns sont les facteurs de risque classiques identifiés dans la population générale (âge, sexe, hypertension artérielle, tabagisme, diabète et hyperlipémie), les autres sont plus spécifiques de l'état urémique notamment certaines altérations métaboliques (phosphocalciques), un état de micro-inflammation et un état de stress oxydant (Drüeke T.B. et al.,2000 ; Goodman WG et al.,2000 ; Mazzafero S et al,1993).

Les maladies cardio-vasculaires étant la première cause de morbimortalité chez les patients en hémodialyse, et les calcifications cardiovasculaires faisant parti de cette entité, il serait pertinent de mener une étude dont le but était de déterminer la prévalence ainsi que les principaux facteurs de risque associés au développement des calcifications valvulaires du cœur et/ou d'au moins un gros tronc artériel chez les hémodialysés. 


\section{Patients et méthodes}

L'étude avait été réalisée dans l'unité d'hémodialyse du service de néphrologie de l'hôpital Aristide Le Dantec (HALD). Il s'agissait d'une étude transversale de type descriptif et analytique de 3 mois allant du $1^{\text {ier }}$ juin 2011 au 31 août 2011.

L'étude portait sur un échantillon de 51 patients de tout âge et de tout sexe confondus qui faisaient régulièrement leurs séances d'hémodialyse depuis au moins 1 an à l'HALD.

\section{Critère d'inclusion :}

-Patients hémodialysés chroniques de plus d'1 an qui avaient présenté une calcification valvulaire du cœur et / ou d'au moins un gros tronc artériel dont le diagnostic avait été fait à l'échographie.

\section{Critères de non inclusion}

N'avaient pas été inclus les patients hémodialysés :

- souffrant d'insuffisance rénale aiguë (IRA)

- n'ayant pas fait l'échographie cardiaque et vasculaire.

Les variables suivantes avaient été étudiées :l'âge, le sexe, la profession, la prise de poids interdialytique, la durée moyenne de mise en dialyse, le nombre de séances réalisées, le nombre d'heures de séance hebdomadaire, la calcémie (VN 85 -100 mg/l), la Phosphorémie (0,45-1,45 $\mathrm{mg} / \mathrm{l})$; la parathormone $(\mathrm{PTH})(\mathrm{VN} 80-200 \mathrm{ng} / \mathrm{l})$, le cholestérol total $(\mathrm{VN}=1$ 2,6g/l), le cholestérol Low Density Lipoprotein (LDL) (VN=0,68-2,4g/l), Hight Density Lipoprotein (HDL), $(\mathrm{VN}=0,35-0,62 \mathrm{~g} / \mathrm{l})$ la triglycéridémie ( $\mathrm{VN}=0,35-1,44 \mathrm{~g} / \mathrm{l})$, le taux d'hémoglobine $(\mathrm{VN}=12-16 \mathrm{~g} / \mathrm{dl})$. L'échographe utilisé était: KONTRON medical, type Imagic agile Rev2. L'échographie avait permis d'étudier les variables comme : le degré de calcification des valves sigmoïdes aortiques, le diamètre de l'aorte et la mesure de l'épaisseur intima/media de l'artère carotide. Les patients qui avaient une calcification cardiaque ou vasculaire étaient des $\mathrm{CCV}(+)$ et ceux qui n'avaient aucune calcification étaient des $\mathrm{CCV}(-)$.

\section{Analyse statistique}

Après le recueil et la collecte des données, nous avions procédé à la saisie informatique puis l'analyse avec épi info 3.5 .3 (version : january 2011).Le test $t$ de Student avait été utilisé pour la comparaison des variables quantitatives et le test $\mathrm{Chi}^{2}$ ou celui de Fisher pour la comparaison des variables qualitatives. Les corrélations entre les données cliniques et biologiques et la présence des calcifications cardiovasculaires avaient été estimées par régression linéaire. Une valeur $\mathrm{p}<0.05$ était considérée comme statistiquement significative. 


\section{Aspect éthique}

Tous les patients recrutés avaient reçu des informations verbales éclairées et avaient délibérément consenti à se soumettre à l'étude.

\section{Résultats}

\section{Données épidémiologiques}

Texte 1 : Sur les 54 patients hémodialysés, 51 avaient été explorés parmi lesquels 39 avaient présenté des calcifications cardiaques et ou vasculaires.

Tableau I. Fréquence des CCV selon l'âge et le sexe (étude comparative)

\begin{tabular}{|l|l|c|c|l|}
\hline \multicolumn{2}{|l|}{ Variables } & $\mathbf{C C V}(+)$ & $\mathbf{C C V}(-)$ & Probabilité \\
\hline \multirow{2}{*}{ Sexe } & Masculin & 13 & 6 & 0.325 \\
\cline { 2 - 4 } & Féminin & 26 & 6 & \\
\hline \multicolumn{2}{|l|}{ Moyenne d'âge } & 50.61 & 41.42 & 0.332 \\
\cline { 1 - 3 } Extrêmes d'âge & $16-82$ & $18-60$ & \\
\hline
\end{tabular}

Il n'existe pas de lien significatif entre le sexe, l'âge et les CCV.

\section{Données cliniques}

Texte 2: dans l'étude les principaux symptômes retrouvés étaient : dyspnée et palpitations qui représentaient chacune $38.5 \%$; la claudication intermittente était de $10.3 \%$. les souffles cardiaques et vasculaires avaient la même fréquence $38,5 \%$.

Tableau II. Comparaison des paramètres généraux

\begin{tabular}{|c|c|c|c|c|}
\hline \multicolumn{2}{|l|}{ Variables } & $\operatorname{CCV}(+)$ & $\operatorname{CCV}(-)$ & Probabilité \\
\hline \multirow{2}{*}{$\begin{array}{l}\text { Nombre d'heures } \\
\text { de } \\
\text { séance } \\
\text { hebdomadaire }\end{array}$} & 12 heures & 22 & 6 & \multirow[t]{2}{*}{0.7} \\
\hline & $\begin{array}{l}\text { Moins de } 12 \\
\text { heures }\end{array}$ & 17 & 6 & \\
\hline \multirow{2}{*}{$\begin{array}{ll}\text { Prise } & \text { de poids } \\
\text { inter } & \text { dialytique } \\
(\mathrm{Kg}) & \end{array}$} & Moyenne & 2.08 & 2.54 & \multirow[t]{2}{*}{0.4} \\
\hline & Extrêmes & $1-4$ & $1-4$ & \\
\hline \multirow{2}{*}{$\begin{array}{l}\text { Durée de mise } \\
\text { en dialyse (mois) }\end{array}$} & Moyenne & 54.08 & 28 & \multirow[t]{2}{*}{0.1} \\
\hline & Extrêmes & $12-352$ & $7-60$ & \\
\hline \multirow{2}{*}{$\begin{array}{l}\text { Nombre de } \\
\text { séances réalisées }\end{array}$} & Moyenne & 600.66 & 259.88 & \multirow[t]{2}{*}{0.2} \\
\hline & Extrêmes & $104-4525$ & $58-520$ & \\
\hline \multirow{2}{*}{$\begin{array}{l}\text { Pression } \\
\text { Artérielle } \\
\text { Systolique (PAS) }\end{array}$} & Moyenne & 140.56 & 143.08 & \multirow[t]{2}{*}{0.8} \\
\hline & Extrêmes & $100-215$ & $100-200$ & \\
\hline \multirow{2}{*}{$\begin{array}{l}\text { Pression } \\
\text { Artérielle } \\
\text { Diastolique } \\
\text { (PAD) }\end{array}$} & Moyenne & 81.48 & 88.75 & \multirow[t]{2}{*}{0.5} \\
\hline & extrêmes & $40-112$ & $60-140$ & \\
\hline
\end{tabular}


Les paramètres généraux ne constituent pas un facteur de risque de survenue des CCV.

Tableau III. Etude comparative des paramètres biologiques

\begin{tabular}{|c|c|c|c|c|}
\hline Variables & & $\mathrm{CCV}(+)$ & $\operatorname{CCV}(-)$ & Probabilité \\
\hline \multirow[t]{5}{*}{ Calcémie (mg/l) } & Augmentée & 6 & 0 & 0.3 \\
\hline & Normale & 32 & 8 & 0.24 \\
\hline & diminuée & 1 & 4 & 0.009 \\
\hline & Moyenne & 95.36 & 81.5 & \\
\hline & Extrêmes & $78-119$ & $60-100$ & \\
\hline \multirow{5}{*}{$\begin{array}{l}\text { Phosphorémie } \\
\text { (mg/l) }\end{array}$} & Augmentée & 9 & 3 & 0.89 \\
\hline & Normale & 26 & 8 & 1.00 \\
\hline & diminuée & 4 & 1 & 1.00 \\
\hline & Moyenne & 42.10 & 40.33 & \\
\hline & Extrêmes & $21-123$ & $23-60$ & \\
\hline \multirow{5}{*}{ PTH (ng/l) } & Augmentée & 7 & & 0.05 \\
\hline & Normale & 14 & & 0.8 \\
\hline & diminuée & 2 & & 0.5 \\
\hline & Moyenne & 451.57 & & \\
\hline & Extrêmes & $18-2083$ & & \\
\hline \multirow[t]{5}{*}{ Hb (g/dl) } & Augmentée & 2 & 2 & 0.23 \\
\hline & Normale & 9 & 2 & 0.73 \\
\hline & diminuée & 28 & 8 & 1.00 \\
\hline & Moyenne & 9.77 & 10.53 & \\
\hline & Extrêmes & $5.40-15.4$ & $8-14$ & \\
\hline \multirow[t]{4}{*}{ HDL(g/l) } & Normale & 32 & 10 & 1.00 \\
\hline & Diminuée & 7 & 2 & 1.00 \\
\hline & Moyenne & 0.50 & 51.16 & \\
\hline & Extrêmes & $0.25-0.98$ & $30-92$ & \\
\hline \multirow{5}{*}{ LDL(g/l) } & Augmentée & 4 & 5 & 0.02 \\
\hline & Normale & 18 & 6 & 1.00 \\
\hline & Diminuée & 17 & 1 & $\mathbf{0 . 0 3 7}$ \\
\hline & Moyenne & 1.27 & 0.61 & \\
\hline & Extrêmes & $0.53-2.01$ & $1.15-2.10$ & \\
\hline \multirow{5}{*}{$\begin{array}{l}\text { Cholestérol total } \\
\text { (g/l) }\end{array}$} & Augmentée & 3 & 4 & 0.04 \\
\hline & Normale & 26 & 7 & 0.73 \\
\hline & Diminuée & 10 & 1 & 0.42 \\
\hline & Moyenne & 1.87 & 2.17 & \\
\hline & Extrêmes & $1.07-2.49$ & $1.50-2.66$ & \\
\hline \multirow{5}{*}{$\begin{array}{l}\text { Triglycérides } \\
(\mathrm{g} / \mathrm{l})\end{array}$} & Augmentée & 2 & 0 & 1.00 \\
\hline & Normale & 33 & 12 & 0.31 \\
\hline & Diminuée & 4 & 0 & 0.56 \\
\hline & Moyenne & 0.96 & 0.87 & \\
\hline & Extrêmes & $0.35-3.07$ & $0.83-1.42$ & \\
\hline
\end{tabular}

Il y a une corrélation entre l'hypocalcémie, le Cholestérol total élevé, LDL(bas et élevé) et PTHi élevé et les CCV. 


\section{Données échographiques}

Tableau IV. Fréquence des calcifications selon le siège et le nombre de valves cardiaques atteintes

\begin{tabular}{|c|c|c|c|}
\hline \multicolumn{2}{|c|}{ Siège } & Nombre & $\%$ \\
\hline \multirow[t]{4}{*}{ Univalvulaire } & Mitrale & 0 & \\
\hline & Tricuspidienne & 0 & \\
\hline & Aortique & 17 & 68 \\
\hline & Pulmonaire & 0 & \\
\hline \multirow[t]{6}{*}{ Bivalvulaire } & Mitro-aortique & 4 & 16 \\
\hline & Mitro-tricuspidienne & 0 & \\
\hline & Mitro-pulmonaire & 0 & \\
\hline & Aorto-pulmonaire & 2 & 8 \\
\hline & Aorto -tricuspidienne & 0 & \\
\hline & Tricuspidienne et pulmonaire & 0 & \\
\hline \multirow[t]{4}{*}{ Trivalvulaire } & Mitrale+ aortique + pulmonaire & 2 & 8 \\
\hline & Mitrale + aortique + tricuspidienne & 0 & \\
\hline & Aortique+pulmonaire+tricuspidienne & 0 & \\
\hline & Mitrale+pulmonaire+tricuspidienne & 0 & \\
\hline \multicolumn{2}{|c|}{ Calcifications légères (degré) } & 17 & 68 \\
\hline \multicolumn{2}{|c|}{ Calcifications modérées (degré) } & 8 & 32 \\
\hline \multicolumn{2}{|l|}{ Total } & 25 & 100.0 \\
\hline
\end{tabular}

Les calcifications aortiques isolées étaient de 17/30, les calcifications mitro-aortiques représentaient $16 \%$ et les calcifications trivalvulaires étaient de $8 \%$.

Tableau V. Fréquence des calcifications et des plaques d'athérome selon des gros troncs artériels atteints $(\mathrm{n} / 30)$

\begin{tabular}{|l|l|l|l|l|l|l|l|}
\hline & \multirow{2}{*}{$\begin{array}{l}\text { Aorte } \\
\text { Abdomi } \\
\text { nale }\end{array}$} & \multicolumn{2}{|c|}{ Carotides } & \multicolumn{2}{c|}{ Iliaques } & \multicolumn{2}{c|}{ fémorales } \\
\cline { 3 - 8 } & & Droite & Gauche & Droite & Gauche & Droite & $\begin{array}{l}\text { Gauch } \\
\text { e }\end{array}$ \\
\hline Calcifications & 6 & 3 & 4 & $\mathbf{1 1}$ & $\mathbf{1 1}$ & $\mathbf{1 2}$ & 6 \\
\hline $\begin{array}{l}\text { Plaques } \\
\text { d'athérome }\end{array}$ & $\mathbf{6}$ & $\mathbf{4}$ & $\mathbf{5}$ & 3 & 3 & 4 & $\mathbf{1 2}$ \\
\hline $\begin{array}{l}\text { Calcifications } \\
\text { +plaques } \\
\text { d'athérome }\end{array}$ & 2 & 3 & 3 & 2 & 2 & 3 & $\mathbf{4}$ \\
\hline Total & $\mathbf{1 4}$ & $\mathbf{1 0}$ & $\mathbf{1 2}$ & $\mathbf{1 6}$ & $\mathbf{1 6}$ & $\mathbf{1 9}$ & $\mathbf{1 9}$ \\
\hline \% & 46.7 & 33.3 & 40 & 53.3 & 53.3 & 63.3 & 63.3 \\
\hline
\end{tabular}

Les calcifications des artères fémorales étaient les plus fréquemment retrouvées soit $63.3 \%$ suivies de $53.3 \%$ des artères iliaques, de l'aorte abdominale $46.7 \%$ et 33.3 à $40 \%$ des artères carotides. 


\section{Discussion}

Parmi les 54 patients qui faisaient régulièrement leurs séances, 51 avaient été recrutés sur la base d'un consentement acquis , 39 avaient présenté des calcifications cardio-vasculaires soit 76.47\%.Benamar AL et al.(2003) avaient rapporté $24,5 \%$ cas de calcifications chez les hémodialysés.

L'étude a trouvé une prédominance féminine soit $66.67 \%$. En 2011, l'étude (Young Son et al.) a montré que 60\% des patients étaient des femmes.Des études réalisées par: Benamar AL et al (2003) et Barreto (2008) ont rapporté une prédominance masculine soit respectivement $66 \%$ et $86 \%$. Bien que le sexe masculin soit un facteur de risque cardio-vasculaire ,ici l'étude ne montre pas de corrélation entre le sexe et les calcifications ce qui serait contraire au résultat de Guerrin et al. (2000).

L'âge moyen des patients était de 50.61 ans avec les extrêmes de 16 82 ans. Les résultats sont différents de ceux de Benamar AL et al. (2000) dont l'étude a montré les extrêmes de 35-55 ans. L'âge n'est pas un facteur de risque de survenue des calcifications cardio-vasculaires. La_Claudication intermittente représentait $10.3 \%$. L'étude de P. Raggi (2002) a trouvé $7 \%$ et un lien entre la claudication et les calcifications.

Soixante-seize virgule neuf pourcent de nos patients avaient une durée de mise en dialyse(DMH). entre 12 et 60 mois. La durée moyenne de mise en hémodialyse était de 54.08 mois. Dans l'observation présente, la D.M.H. n'est pas un facteur de risque du développement des calcifications cardiovasculaires. Benamar AL et al. (2000) et D .V. Barrreto et al.(2008) n'ont pas, trouvé de corrélation entre la D.M.H. et la survenue des calcifications cardiovasculaires. La D.M.H. dans l'observation de D .V. Barrreto et al.(2008) est de $36.3 \pm 22.3$ mois.

Cinquante-six virgule quatre pourcent de nos patients ont fait un nombre de séances compris entre 104 et 520. La moyenne des séances réalisées était de 600.66 séances avec des extrêmes de 104 à 4525 .

Dans l'étude il n'y a pas de corrélation entre le nombre de séances réalisées et la présence des calcifications cardio-vasculaires.

La calcémie était normale chez $82 \%$ des patients , $2.6 \%$ avaient une hypocalcémie et $15.4 \%$ avaient une hypercalcémie. La moyenne était de 95.36 $\mathrm{mg} / \mathrm{l}$.

Une corrélation entre l'hypocalcémie et la présence des calcifications cardio-vasculaires a été observée. Ce qui concorde avec les données de Benamar AL. et al. (2000) et Goodman WG. (2004)

L'étude a noté $46.1 \%$ cas de LDL normal, $43.6 \%$ de LDL bas, et $10.3 \%$ LDL élevé . La moyenne était de $1.27 \mathrm{~g} / \mathrm{l}$.

Le taux sanguin de LDL (bas et augmenté) est un facteur de risque du développement des calcifications cardiovasculaires $(p<0.05)$. L'étude de 2011 a fait constaté la même observation (Young K. Son et al). 
Dans l'étude $66.67 \%$ des patients avaient un taux normal de cholestérolémie totale ; $25.64 \%$ avaient un taux en dessous de la normale et $7.69 \%$ étaient en hypercholestérolémie.

La moyenne était de $1.87 \mathrm{~g} / \mathrm{l}$. Il existe une différence statistiquement significative entre le cholestérol total élevé et les CCV. Dans l'étude de Young K. Son et al (2011), ce constat est le même alors que Benamar AL. et al. (2000) ont rapporté une observation contraire.

Parmi les 23 patients qui avaient fait le dosage sanguin de la PTHi, $60.67 \%$ avaient une valeur normale, $8.7 \%$ avaient un taux en dessous de $21 \mathrm{ng} / \mathrm{l}$ et $30.43 \%$ avaient un taux supérieur à la normale. La moyenne était de 451.57 $\mathrm{ng} / \mathrm{l}$.

Dans l'observation il existe une différence statistiquement significative entre le taux élevé de PTHi et la présence des calcifications cardio-vasculaires. K/Doqi (2003) et Wang AY, Wang M, Woo J. (2003) ont fait remarquer que le taux de PTHi n'est pas un facteur de risque associé aux CCV.

Les calcifications valvulaires étaient de $64.1 \%$ et vasculaires 76.9\%.L'étude de Sharzad Ossareh et al. (2011) a fait observer 50\% de calcifications valvulaires et $60 \%$ vasculaires.

Selon le nombre de valves atteintes les calcifications Univalvulaires représentaient $68 \%$, bivalvulaires $24 \%, 8 \%$ trivalvulaires.Wang AY, Wang M, Woo J. (2003) ont rapporté $45 \%$ de calcifications au niveau de l'anneau mitral, $34 \%$ au niveau des valves aortiques et $21 \%$ cas de calcifications mitro-aortiques ;Strauman (1992) a obtenu $40 \%$ de calcifications mitrales, $55 \%$ aortiques et $5 \%$ mitro-aortiques.

Les calcifications des artères fémorales représentaient $63.3 \%$ suivies des calcifications des artères iliaques $53.3 \%$ et de l'aorte abdominale 46.7\%.Benamar AL. et al. (2000) ont trouvé $18 \%$ de calcifications au niveau de l'aorte lombaire et $12.8 \%$ au niveau des artères iliaques.

\section{Conclusion}

Les CCV sont fréquentes chez les hémodialysés et constituent l'une des causes de l'augmentation de la morbi-mortalité.

Dans l'étude, les facteurs de risque liés au développement des calcifications cardio-vasculaires étaient: l'hypocalcémie, l'hypo et l'hypercholestérolémie à LDL, l'hyper cholestérolémie totale et le taux sanguin de PTHi augmenté. .

La réalisation des explorations cardiovasculaires chez tous les IRC avant et pendant la dialyse permet le diagnostic précoce.

Les techniques d'imagerie plus sensibles permettent de déterminer précocement et quantitativement les $\mathrm{CCV}$. 
Le suivi collégial est nécessaire pour les patients ayant des calcifications valvulaires et/ou vasculaires. des CCV.

La correction des facteurs de risque permet de prévenir la survenue

\section{Conflit d'intérêt}

Les auteurs déclarent qu'il n'y a aucun conflit d'intérêt lié à cette étude.

\section{References:}

1. Benamar, A L, Rhou, H, Guerraoui, MH et al. (2000). Calcifications cardiovasculaires chez l'hémodialysé chronique. Prévalence et facteurs de risque $24: 143-14$.

2. Daniela Veit Barreto, Fellype de Carvalho Barreto,Aluízio Barbosa de Carvalho, et al. (2008) Association of Changes in Bone Remodeling and Coronary Calcificationin Hemodialysis Patients: A Prospective Study. J.K. Diseases, Vol 52, No 6: 1139-1150

3. Defrancisco AM, Ellis HA, Owen JP et al. (1985).Parathyroidectomy in chronic renal failure. Q J Med, 55, 289-315.

4. Drüeke T.B.,Touam M., Thornley-Brown D. et al. (2000) : Calcifications extra-osseuses chez le patient insuffisant rénal chronique. Actualités Néphrologiques Jean Hamburger, Hôpital Necker Paris, Flammarion, p. $53-81$

5. Goodman WG, Goldin J, Kuizon BD, et al. (2000). Coronaryarterycalcification in young adults with end stage renal disease who are undergoing dialysis. N Engl J Med ; 20 : 1478-83.

6. Goodman WG. (2004). Importance of hyperphosphataemia in the cardio-renal axis. Nephrol Dial Transplant;19:14-18.

7. Guerin AP, London GM, Marchais SJ, Metivier F. (2000). Arterial stiffening and vascular calcifications in end-stage renal disease. Nephrol Dial Transplant; ;15(7):1014-21

8. K/Doqi (2003). Clinical Practice Guidelines for Bone Metabolism and Disease in Chronic Kidney Disease. Am J Kidney Dis; 42:S1-S255.

9. Kalantar-Zadeh K, Kuwae N, Redigor DL, et al. (2006). Survival predictability of time-varying indicators of bone disease in maintenance hemodialysis patients.Kidney Int.;70:771-80

10. Mazzafero S, Coen G, Bandini S, et al. (1993). Role of aging, chronic renal failure and dialysis in the calcification of mitral annulus. Nephrol Dial Transplant ; $8: 335-40$.

11. Paolo Raggi,, Amy Boulay, Scott Chasan-Taber, Naseem Amin, et al. (2002). Cardiac Calcification in Adult Hemodialysis Patients A Link Between End-Stage Renal Disease and Cardiovascular Disease? Journal of the American College of Cardiology 39: 695-701. 
12. Shahrzad Ossareh (2011)Vascular Calcification in Chronic Kidney DiseaseMechanisms and Clinical Implications Iranian Journal of Kidney Diseases ;5:285-99.

13. Straumann E, Meyer B, Mistelli et al. (1992)Aortic and mitral valve disease in patients with end stage renal faillure on long term haemodialysis, Heart ; 67 ; $236-9$

14. Wang AY, Wang M, Woo J. (2003)Cardiac Valve Calcification as an Important Predictor for All- Cause Mortality and Cardiovascular Mortality in Long-Term Peritoneal Dialysis Patients: A Prospective Study. J Am Soc Nephrol 13: 159-168.

15. Young K. Son, Su M. Lee, Seong E. Kim, et al. (2011). Association Between Vascular Calcification Scores on Plain Radiographs and Fatty Acid Contents of Erythrocyte Membrane in Hemodialysis PatientsJournal of Renal Nutrition, 1053 : 1-9 